\title{
Analytic Variations on the Airy Distribution ${ }^{1}$
}

\author{
P. Flajolet ${ }^{2}$ and G. Louchard ${ }^{3}$
}

\begin{abstract}
The Airy distribution (of the "area" type) occurs as a limit distribution of cumulative parameters in a number of combinatorial structures, like path length in trees, area below walks, displacement in parking sequences, and it is also related to basic graph and polyomino enumeration. We obtain curious explicit evaluations for certain moments of the Airy distribution, including moments of orders $-1,-3,-5$, etc., as well as $+\frac{1}{3},-\frac{5}{3},-\frac{11}{3}$, etc. and $-\frac{7}{3},-\frac{13}{3},-\frac{19}{3}$, etc. Our proofs are based on integral transforms of the Laplace and Mellin type and they rely essentially on "non-probabilistic" arguments like analytic continuation. A byproduct of this approach is the existence of relations between moments of the Airy distribution, the asymptotic expansion of the Airy function $\operatorname{Ai}(z)$ at $+\infty$, and power symmetric functions of the zeros $-\alpha_{k}$ of $\operatorname{Ai}(z)$.
\end{abstract}

Key Words. Brownian excursion area, Airy function, Parking problem, Linear probing hashing.

Introduction. For probabilists, the Airy distribution considered here is nothing but the distribution of the area under the Brownian excursion. The name is derived from the connection between Brownian motion and the Airy function, a fact discovered around 1980 by several authors; see [16] and [20]. For combinatorialists and theoretical computer scientists, this Airy distribution (of the "area type") arises in a surprising diversity of contexts like parking allocations, hashing tables, trees, discrete random walks, mergesorting, etc.

The most straightforward description of the Airy distribution is by its moments themselves defined by a simple nonlinear recurrence. We follow here the notations and the normalization of [11].

DEFINITION 1. The Airy distribution (of the "area" type) is the distribution of a random variable $\mathcal{A}$ whose moments are

$$
\mu_{r} \equiv E\left(\mathcal{A}^{r}\right)=\frac{-\Gamma\left(-\frac{1}{2}\right)}{\Gamma((3 r-1) / 2)} \Omega_{r}, \quad r \geq 1,
$$

where the "Airy constants" $\Omega_{r}$ are determined by the quadratic recurrence

$$
\Omega_{0}=-1, \quad 2 \Omega_{r}=(3 r-4) r \Omega_{r-1}+\sum_{j=1}^{r-1}\left(\begin{array}{l}
r \\
j
\end{array}\right) \Omega_{j} \Omega_{r-j} \quad(r \geq 1) .
$$

\footnotetext{
${ }^{1}$ This research was partially supported by the IST Programme of the EU under Contract Number IST-199914186 (ALCOM-FT).

2 INRIA, Domaine de Voluceau-Rocquencourt, BP 105, F-78153 Le Chesnay Cedex, France. Philippe.Flajolet@inria.fr.

${ }^{3}$ Département d'Informatique, Université Libre de Bruxelles, CP 212, Boulevard du Triomphe, B-1050 Bruxelles. louchard@ulb.ac.be.
}

Received June 6, 2000; revised February 17, 2001. Communicated by R. Kemp and H. Prodinger. Online publication August 28, 2001. 
Table 1. A table of the Airy constants $\Omega_{r}$ and of the Airy moments $\mu_{r}$.

\begin{tabular}{lcccccccc}
\hline$r$ & 0 & 1 & 2 & 3 & 4 & 5 & 6 & 7 \\
\hline$\Omega_{r}$ & -1 & $\frac{1}{2}$ & $\frac{5}{4}$ & $\frac{45}{4}$ & $\frac{3315}{16}$ & $\frac{25,425}{4}$ & $\frac{18,635,625}{64}$ & $\frac{18,592,875}{1}$ \\
$\mu^{(r)}$ & 1 & $\sqrt{\pi}$ & $\frac{10}{3}$ & $\frac{15}{4} \sqrt{\pi}$ & $\frac{884}{63}$ & $\frac{565}{32} \sqrt{\pi}$ & $\frac{662,600}{9009}$ & $\frac{19,675}{192} \sqrt{\pi}$ \\
\hline
\end{tabular}

The normalized random variable

$$
\mathcal{B}=\frac{\mathcal{A}}{\sqrt{8}}
$$

is called the "Brownian excursion area" (BEA).

The first few values of the $\Omega_{r}$ and of moments $\mu_{r}$ are given in Table 1 . We shall see later that the Airy distribution is uniquely determined by its moments and that it admits a continuous density $w(x)$. The definition by moments as given above is natural from the point of view of discrete mathematics, where the quadratic recurrence of moments usually appears as a more or less distant echo of some simple combinatorial tree decomposition. The Airy distribution arises in the following contexts:

1. In probability theory, $\mathcal{B}=\mathcal{A} / \sqrt{8}$ is the distribution of the area $\int_{0}^{1} X(t) d t$ under the Brownian excursion $X(t)(X(0)=X(1)=0, X(t) \geq 0)$. See Louchard's works [16], [17]. The Airy distribution occurs in related problems relative to the analysis of dynamic data structures (like stacks under a Markovian model and priority queues under Knuth's model), to the busy period of an $M / G / 1$ queue, and to the area of various classes of polyominoes; see [12] on the dynamic aspects and [8] for applications to polyominoes.

2. Accordingly, $\mathcal{A}$ is, up to normalization, the limit distribution of the area under discrete excursions, like the Bernoulli excursion [17], [22] (the probabilities of \pm 1 jumps are $1 / 2$ ) or the Poisson excursion (each jump is $-1+Y$ where $Y$ is Poisson(1) distributed), where the latter was introduced by Spencer [21] in order to model traversals of random connected graphs.

3. Path length in trees (that is, the sum of distances from the root to all the nodes) is asymptotically Airy distributed in Catalan trees as well as in other combinatorial families of trees that are defined by a finite set of allowed node degrees. This fact was proved by Takács [22]-[24] whose results apply also to trees determined by branching processes and conditioned by the size of the total progeny.

4. The number of inversions in a random Cayley tree of large size is Airy distributed in the limit [11].

5. The total displacement of a random parking sequence or equivalently the construction cost of a hashing table under the linear probing strategy is Airy distributed in the limit. See [11] and especially the work of Knuth [15] for a perspective.

6. The Airy constant $\Omega_{r}$ appears in the asymptotic enumeration of labelled connected graphs with $n$ vertices and $n+r-1$ edges, an intriguing fact that points to deep connections between random graphs and random walks [11], [13], [15], [21], [27] 
7. As implied by recent results of Chassaing and Marckert [5], breadth first search traversal of random trees has a cumulated cost that is asymptotically Airy distributed. Chassaing and Marckert's study also provides interesting connections with empirical processes and the additive coalescent of Aldous and Pitman fame [2].

Scope. In this note we examine analytic properties of the Airy distribution. The most curious fact, perhaps, is that certain moments of fractional and of negative orders can be explicitly evaluated in terms of $\pi$ and of the gamma function evaluated at one-third. The paper is also meant as a partial survey of fundamental properties of the Airy distribution and the somewhat radical point of view adopted here is that of an analyst's rather than of a probabilist's. In the perspective of discrete mathematics, the logical chain is as follows:

- First, decomposable discrete models like area under a Bernoulli excursion or total displacement in parking sequences lead rather directly to functional equations over bivariate generating functions, for instance,

$$
E(z, q)=\frac{z}{1-E(q z, q)}, \quad \frac{\partial}{\partial z} F(z, q)=F(z, q) \cdot \frac{F(z, q)-q F(q z, q)}{1-q},
$$

for area and displacement; see [9] and [11], [15], respectively.

- Second, (univariate) generating functions of factorial moments satisfy relations that are deduced from bivariate functional equations by differentiation and specialization at $q=1$. Asymptotic forms of the moments then come out naturally through singularity analysis of the univariate functional relations: this is the method of "pumping moments" exemplified by [11], [14], [16], [24]. A number of situations then lead rather elementarily to the moment recurrence of Definition 1 above.

- Third, the moments determine a probability distribution that is (by moment convergence theorems) the limit law of the combinatorial parameter under consideration.

Following Louchard and Takács [16], [24], we feel that there may be interest in drawing conclusions that take off from a direct definition by moments ((1) and (2)) and proceed from there straightforwardly using basic analysis.

1. The Airy Distribution: Generating Functions. The term "Airy distribution" comes from the relation between the fundamental constants $\Omega_{r}$ and the Airy function $\operatorname{Ai}(z)$ (a solution of $y^{\prime \prime}-z y=0$ ), or, alternatively, with Bessel functions of orders that are multiples of one-third. We recall here the standard definitions as found in [1]:

$$
\begin{aligned}
I_{\nu}(z) & =\left(\frac{z}{2}\right)^{v} \sum_{k=0}^{\infty} \frac{\left(z^{2} / 4\right)^{k}}{k ! \Gamma(v+k+1)}, \\
K_{v}(z) & =\frac{\pi}{2 \sin v \pi}\left(I_{-v}(z)-I_{v}(z)\right), \\
\operatorname{Ai}(z) & =\frac{1}{\pi} \int_{0}^{\infty} \cos \left(\frac{1}{3} t^{3}+z t\right) d t
\end{aligned}
$$




$$
\begin{aligned}
& =\frac{1}{\pi}\left(\frac{z}{3}\right)^{1 / 2} K_{1 / 3}\left(\frac{2 z^{3 / 2}}{3}\right), \\
{ }_{2} F_{0}[\alpha, \beta ; z] & =1+\frac{\alpha \cdot \beta}{1 !} z+\frac{\alpha(\alpha+1) \cdot \beta(\beta+1)}{2 !} z^{2}+\cdots,
\end{aligned}
$$

where the last relation (7) is to be taken in the sense of formal power series.

Proposition 1 [16], [24]. The Airy constants $\Omega_{r}$ are characterized by any of the following expansions:

$$
\begin{gathered}
-\frac{I_{2 / 3}(z / 3)}{I_{-1 / 3}(z / 3)} \underset{z \rightarrow+\infty}{\sim} \sum_{r=0}^{\infty} \Omega_{r} \frac{z^{-r}}{r !} \\
3 \frac{d}{d z} \log K_{1 / 3}\left(\frac{z}{3}\right)_{z \rightarrow+\infty}^{\sim}-\frac{1}{z}+\sum_{r=0}^{\infty} \Omega_{r} \frac{(-z)^{-r}}{r !} \\
\frac{\operatorname{Ai}^{\prime}(z)}{\operatorname{Ai}(z)} \underset{z \rightarrow+\infty}{\sim} \sum_{r=0}^{\infty} \frac{\Omega_{r}}{2^{r}} \frac{(-1)^{r} z^{-(3 r-1) / 2}}{r !}, \\
\sum_{r \geq 0} \Omega_{r} \frac{w^{r}}{r !}=-\frac{\Phi_{2 / 3}(w)}{\Phi_{-1 / 3}(w)}, \quad \Phi_{v}(w)={ }_{2} F_{0}\left[\frac{1}{2}+v, \frac{1}{2}-v ;\left(\frac{3}{2} w\right)\right] .
\end{gathered}
$$

PROOF (SKETCH). The recurrence (2) for the Airy constants $\Omega_{r}$ translates into a differential equation of the Riccati type for the associated generating function whose solution is the logarithmic derivative of the solution of a second-order differential equation (as seen via the usual linearization transformation of Riccati equations). This provides the Bessel-Airy connection; see [11] for details in the case of (8), cases (9) and (10) being similar. Finally, formula (11), to be understood as a formal power series equivalence, derives directly from (10) combined with the classical asymptotic expansion of the Airy function [1, Entry 10.4.59],

$$
\begin{gathered}
\operatorname{Ai}(z) \sim \frac{1}{2} \pi^{-1 / 2} z^{-1 / 4} e^{-\zeta} \sum_{k=0}^{\infty}(-1)^{k} c_{k} \zeta^{-k}, \\
\zeta=\frac{2}{3} z^{3 / 2}, \quad c_{k}=\frac{\Gamma\left(3 k+\frac{1}{2}\right)}{54^{k} k ! \Gamma\left(k+\frac{1}{2}\right)}, \quad z \rightarrow+\infty,
\end{gathered}
$$

that is valid for large positive arguments.

It is interesting to note that the representations (8)-(10) are asymptotic series when the variable $z$ tends to $+\infty$. There is a nonalternating representation in terms of Bessel- $I$ functions (that grow exponentially fast in (8)), as well as an alternating form in terms of Bessel- $K$ functions (9), or equivalently in terms of the Airy function (in (10) that decays exponentially). It is on the better conditioned Airy representations (10) that the rest of our treatment is based. In contrast to the defining quadratic recurrence, the hypergeometric 
formula (11) implies a linear recurrence [16] on the Airy coefficients $\Omega_{r}$ that, although of computational interest, does not tend to arise directly in combinatorial applications:

$$
18^{r} \Omega_{r}=\frac{12 r}{6 r-1} \frac{\Gamma\left(3 r+\frac{1}{2}\right)}{\Gamma\left(r+\frac{1}{2}\right)}-\sum_{k=1}^{r-1}\left(\begin{array}{l}
r \\
k
\end{array}\right) \frac{\Gamma\left(3 k+\frac{1}{2}\right)}{\Gamma\left(k+\frac{1}{2}\right)} 18^{r-k} \Omega_{r-k} .
$$

Furthermore, elementary manipulations of divergent series applied to (11) yield an asymptotic estimate for $\Omega_{r}$, hence for $\mu_{r}$,

$$
\mu_{r} \sim 2^{1 / 2} 3 r\left(\frac{2 r}{3 e}\right)^{r / 2},
$$

as noted by Takács. (Janson et al. discuss the history of this expansion on pp. 262-263 of [13], with contributions by Wright, Stepanov, Bagaev, Dmitriev, Meertens, and Vobly̌̆.) It results in particular that the Carleman condition [4]

$$
\sum_{r}\left(\mu_{r}\right)^{-1 / r}=+\infty
$$

is satisfied, so that the Airy distribution is uniquely determined by its moments.

2. The Airy Distribution: Laplace Transform and Density. The relation between Airy coefficients and moments of the Airy distribution involves products and quotients of factorials that, at generating functions level, are well known to correspond to direct and inverse Laplace transforms. Let $w(x), W(x)$ be the density and the distribution function of the Airy distribution,

$$
W(x):=\operatorname{Pr}\{\mathcal{A} \leq x\}, \quad w(x)=\frac{d}{d x} W(x),
$$

with $G(y)$ the corresponding moment generating function,

$$
G(y)=\sum_{r \geq 0} \mu_{r} \frac{(-y)^{r}}{r !}=E\left[e^{-y \mathcal{A}}\right]=\int_{0}^{\infty} e^{-y t} d W(t) .
$$

See Figure 1 for a display of $\operatorname{Ai}(x)$ and $w(x)$. We have:

Proposition 2 [16], [24]. The Airy distribution function satisfies the double Laplace transform relation:

$$
\frac{1}{\sqrt{2 \pi}} \int_{0}^{\infty}\left(e^{-z y}-1\right) G\left(2^{-3 / 2} y^{2 / 3}\right) \frac{d y}{y^{3 / 2}}=2^{1 / 3}\left(\frac{\operatorname{Ai}^{\prime}\left(2^{1 / 3} z\right)}{\operatorname{Ai}\left(2^{1 / 3} z\right)}-\frac{\mathrm{Ai}^{\prime}(0)}{\operatorname{Ai}(0)}\right)
$$

The moment generating function and the density of the Airy distribution are given by

$$
\begin{aligned}
G\left(2^{-3 / 2} y\right) & =\sqrt{2 \pi} y \sum_{k=0}^{\infty} \exp \left(-\alpha_{k} y^{2 / 3} 2^{-1 / 3}\right), \\
2^{3 / 2} w\left(2^{3 / 2} x\right) & =\frac{2 \sqrt{6}}{x^{2}} \sum_{k=1}^{\infty} e^{-v_{k}} v_{k}^{2 / 3} U\left(\frac{-5}{6}, \frac{4}{3} ; v_{k}\right), \quad v_{k}=\frac{2 \alpha_{k}^{3}}{27 x^{2}} .
\end{aligned}
$$




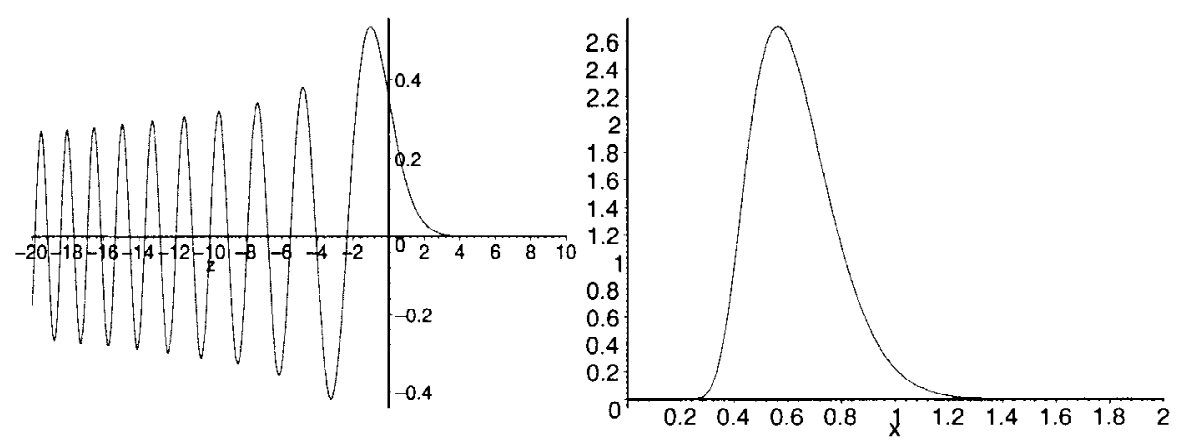

Fig. 1. Plots of the Airy function $\operatorname{Ai}(x)$ (left) for $x=-20 \cdots 10$, and of the density of the Brownian excursion area $2^{3 / 2} w\left(2^{3 / 2} x\right)$ (right) for $x=0 \cdots 2$.

There, the quantities $-\alpha_{k}$ are the zeros of the Airy function $\operatorname{Ai}(z)$ and $U(a, b ; z)$ is the confluent hypergeometric function (17).

We recall that the confluent hypergeometric function $U$ is defined as

$$
U(a, b ; z)=\frac{\pi}{\sin \pi b}\left(\frac{{ }_{1} F_{1}[a, b ; z]}{\Gamma(1+a-b) \Gamma(b)}-\frac{z^{1-b}{ }_{1} F_{1}[1+a-b, 2-b ; z]}{\Gamma(a) \Gamma(2-b)}\right),
$$

where ${ }_{1} F_{1}[a ; b ; z]$ is the hypergeometric function (Kummer's function)

$$
{ }_{1} F_{1}[a ; b ; z]=1+\frac{a}{b} \frac{z}{1 !}+\frac{a(a+1)}{b(b+1)} \frac{z^{2}}{2 !}+\cdots .
$$

For comparison with standard results in the literature, the data in (15) and (16) are expressed in terms of the moment generating function $G_{\mathcal{B}}$ and density $w_{\mathcal{B}}$ of the Brownian excursion area $\mathcal{B}=\mathcal{A} / \sqrt{8}$ that satisfy

$$
G_{\mathcal{B}}(y)=G\left(y 2^{-3 / 2}\right), \quad w_{\mathcal{B}}(x)=2^{3 / 2} w\left(2^{3 / 2} x\right) .
$$

PROOF (SKETCH). Equations (14) and (15) are due to Louchard, while (16) is due to Takács. Relation (14) follows formally by application of two successive Laplace transforms to the expression (9). Then (15) results from a residue computation of the inverse Laplace integral of (14). Finally, relation (16) comes from the fact that the inverse Laplace transform of $e^{-x^{2 / 3}}$ is a confluent hypergeometric function.

Thus, the Airy density is essentially a double inverse Laplace transform of the logarithmic derivative of the Airy function. We now briefly discuss its tails. The left tail is of an exponential type and it is expressed in terms of the zero of Airy's function that is of smallest modulus.

THEOREM 1 (Left Tail of the Airy Distribution [16]). Let $-\alpha_{1} \doteq-2.3381074104$ be the first Airy zero. As $x \rightarrow 0^{+}$, one has

$$
2^{3 / 2} w\left(2^{3 / 2} x\right) \sim e^{-2 \alpha_{1}^{3} /\left(27 x^{2}\right)}\left(\frac{8}{35} \frac{\alpha_{1}^{9 / 2}}{x^{5}}-\frac{48}{35} \frac{\alpha_{1}^{3 / 2}}{x^{3}}-\frac{11664}{35} \frac{\alpha_{1}^{-3 / 2}}{x}+\cdots\right) .
$$


PROOF. When $x$ tends to 0 , the first term in (16) corresponding to $\alpha_{1}$ dominates exponentially all the other ones. The expansion then comes out as an immediate consequence of Takács's expression (16) and of the known asymptotic expansion of the confluent hypergeometric function (see Entry 13.5.2 on p. 508 of [1]):

$$
U(a, b, z) \sim z^{-a}\left(\sum_{n=0}^{\infty} \frac{(a)_{n}(1+a-b)_{n}}{n !}(-z)^{-n}\right), \quad z \rightarrow+\infty .
$$

The first two terms of the expansion of $w$ were derived by Louchard in [16] (with his constant in the second asymptotic term that needs to be corrected) using a saddle point analysis of the integral that represents the inverse Laplace transform of $G(y)$. The easy derivation given here builds upon the later formula of Takács (16) and has the advantage of being fully explicit thanks to (18).

The right tail seems to be less precisely quantified at the moment. As kindly pointed to us by Marc Yor (private communication, December 1999), what is available is the following recent estimate of [6] obtained by probabilistic methods.

THEOREM 2 (Right Tail of the Airy Distribution [6]). As $y \rightarrow+\infty$, one has

$$
\log \operatorname{Pr}\left(\frac{\mathcal{A}}{\sqrt{\forall}}>y\right) \sim-6 y^{2} .
$$

In the perspective of our paper, it would be of interest to be able to get access to the right tails by purely analytic methods.

Properties of the Airy Zeros. Airy zeros play an important rôle in what follows and we briefly summarize here some relevant facts. The Airy function is known to have zeros confined to the negative real axis. The sequence starts with

$$
-\alpha_{1} \doteq-2.33, \quad-\alpha_{2} \doteq-4.08, \quad-\alpha_{3} \doteq-5.52, \quad-\alpha_{4} \doteq-6.78 .
$$

From the oscillating form of $\operatorname{Ai}(x)$ along the negative real axis [1, Entry 10.4.60],

$$
\begin{aligned}
& \operatorname{Ai}(-z) \sim \pi^{-1 / 2} z^{-1 / 4}\left(\sin Z \sum_{k=0}^{\infty}(-1)^{k} c_{2 k} \zeta^{-2 k}-\cos Z \sum_{k=0}^{\infty}(-1)^{k} c_{2 k+1} \zeta^{-2 k-1}\right), \\
& \text { (19) } \quad \zeta=\frac{2}{3} z^{3 / 2}, \quad Z=\zeta+\frac{\pi}{4}, \quad c_{k}=\frac{\Gamma\left(3 k+\frac{1}{2}\right)}{54^{k} k ! \Gamma\left(k+\frac{1}{2}\right)}, \quad z \rightarrow+\infty,
\end{aligned}
$$

it is not hard to see that the $k$ th zero admits a full asymptotic expansion in descending powers of $k$.

LEMMA 1. The Airy zeros admit an asymptotic expansion of the form:

$$
\alpha_{k} \sim \rho k^{2 / 3}\left(1+\sum_{j=1}^{\infty} \frac{a_{j}}{k^{j}}\right) \quad \text { with } \rho=\left(\frac{3 \pi}{2}\right)^{2 / 3} .
$$


This relation is Entry 10.4.94 of [1] and the expansion starts as

$$
\alpha_{k} \sim \rho k^{2 / 3}\left(1-\frac{1}{6 k}-\frac{\rho^{3}-15}{144 \rho^{3} k^{2}}-\frac{\rho^{3}-45}{1296 \rho^{3} k^{3}}-\cdots\right) .
$$

Thus, the absolute values $\alpha_{k}$ of the Airy zeros grow like $k^{2 / 3}$, so that the sum in (16) expressing the Airy density is rapidly converging. This fact guarantees in particular the existence of the density function $w(x)$.

3. The Airy Distribution: Mellin Transforms. In this section we examine the effect of the classical Mellin transform on the various generating functions of the previous section. The Mellin transform of a function $f(t)$ defined on $(0,+\infty)$ is by definition

$$
\mathcal{M}[f]=f^{*}(s):=\int_{0}^{\infty} f(t) t^{s-1} d t .
$$

If $f(t)$ is the probability density of a random variable $X$, then $f^{*}(s)$ is nothing but a moment of $X$ :

$$
f^{*}(s)=\mathbf{E}\left(X^{s-1}\right) .
$$

As a consequence, any explicit value of the Mellin transform of a probability density provides an explicit value of the corresponding moment. This is the strategy used in the remainder of the paper in order to evaluate nonstandard moments of the Airy distribution.

We first recall some operational principles of Mellin transforms. By linearity, the definition of the Mellin transform implies transformation rules for "harmonic sums" and "harmonic integrals":

$$
\begin{gathered}
F(t)=\sum_{k} \lambda_{k} f\left(\mu_{k} t\right) \quad \Longrightarrow \quad F^{*}(s)=\left(\sum_{k} \lambda_{k} \mu_{k}^{-s}\right) \cdot f^{*}(s), \\
F(t)=\int_{0}^{\infty} \lambda(\xi) f(\xi t) d \xi \quad \Longrightarrow F^{*}(s)=\lambda^{*}(1-s) \cdot f^{*}(s) .
\end{gathered}
$$

We refer to the survey paper [10] and to the book by Wong [26] for detailed validity conditions.

There is also a well-known correspondence—called the "mapping property"-that fares both ways and relates the asymptotic expansions of an original function $f(t)$ at 0 and $+\infty$ and poles of the transform $f^{*}(s)$ in a left and right half-plane, respectively; see [7], [10], and [26] for details.

In what follows, an essential rôle is played by what may be called the "root zeta function" of the Airy function. This function is defined by

$$
\Lambda(s):=\sum_{k=1}^{\infty}\left(\alpha_{k}\right)^{-s} \quad\left(\Re(s)>\frac{3}{2}\right)
$$


where the sum is a priori defined and analytic for $\Re(s)>\frac{3}{2}$, given the growth of the $\alpha_{k}$ described by (20). Furthermore, since $\alpha_{k}$ admits a complete asymptotic expansion, $\Lambda(s)$ can be in fact continued as a meromorphic function in the whole of the complex plane. The continuation property results from a classical process of inserting "convergence terms" in a divergent series. For instance, the identity

$$
\Lambda(s)=\sum_{k \geq 1}(3 \pi k / 2)^{-2 s / 3}+\sum_{k \geq 1}\left(\left(\alpha_{k}\right)^{-s}-(3 \pi k / 2)^{-2 s / 3}\right)
$$

initially valid for $\Re(s)>\frac{3}{2}$ extends by analytic continuation to $\Re(s)>0$, where the first term in (26) is $(3 \pi / 2)^{-2 s / 3} \zeta(s)$ with $\zeta(s)$ the Riemann zeta function and the second term has summands that are $O\left(k^{-2 / 3 s-1}\right)$ because of (20). This construction generalizes.

LEMMA 2. The root zeta function $\Lambda(s)$ is meromorphic in the whole of $\mathbb{C}$. For any integer $q \geq 0$, it admits an expansion with convergence terms, valid in $\Re(s)>-\frac{3}{2} q$, of the form

$$
\begin{aligned}
\Lambda(s)= & \sum_{k \geq 1}\left(\frac{1}{\left(\alpha_{k}\right)^{s}}-\frac{1}{\rho^{s} k^{2 s / 3}}\left(g_{0}+\cdots+\frac{g_{q}(s)}{k^{q}}\right)\right) \\
& +\rho^{-s}\left(\sum_{j=0}^{q} g_{j}(s) \zeta\left(\frac{2}{3} s+j\right)\right) .
\end{aligned}
$$

The polynomials $g_{j}(s)$ are defined in terms of the asymptotic expansion (20) by

$$
g_{0}=1, \quad 1+\sum_{j=1}^{\infty} \frac{g_{j}(s)}{k^{j}} \sim\left(1+\sum_{j=1}^{\infty} \frac{a_{j}}{k^{j}}\right)^{-s} .
$$

In particular, from (21), one finds mechanically

$$
g_{0}(s)=1, \quad g_{1}(s)=\frac{1}{6} s, \quad g_{2}(s)=\frac{1}{144 \rho^{3}} s\left(2 s \rho^{3}+3 \rho^{3}-15\right), \ldots,
$$

with the corresponding continuations of $\Lambda(s)$ :

$$
\begin{aligned}
& \Lambda(s)=\sum_{k} \frac{1}{\alpha_{k}^{s}} \quad\left(\Re(s)>\frac{3}{2}\right), \\
& \Lambda(s)=\sum_{k}\left(\frac{1}{\alpha_{k}^{s}}-\frac{1}{\rho^{s} k^{2 s / 3}}\right)+\frac{1}{\rho^{s}} \zeta\left(\frac{2}{3} s\right) \quad(\Re(s)>0), \\
& \Lambda(s)=\sum_{k}\left(\frac{1}{\alpha_{k}^{s}}-\frac{1}{\rho^{s} k^{2 s / 3}}\left[1+\frac{s}{6 k}\right]\right)+\frac{1}{\rho^{s}}\left[\zeta\left(\frac{2}{3} s\right)+\frac{s}{6} \zeta\left(\frac{2}{3} s+1\right)\right] \quad\left(\Re(s)>-\frac{3}{2}\right) .
\end{aligned}
$$

Lemma 2 implies that $\Lambda(s)$ has simple poles at a subset of the points

$$
s=\frac{3}{2}, 0,-\frac{3}{2},-3,-\frac{9}{2}, \ldots,
$$


and is of polynomial growth at $\pm i \infty$ in any right half-plane. We shall verify later (see Section 5 and (43)) that $\Lambda(0), \Lambda(-3), \Lambda(-6), \ldots$ are all finite, so that $\Lambda$ is in fact analytic at these points. We can now state:

PROPOSITION 3. The following Mellin transform formulae hold:

$$
\begin{aligned}
\mathcal{M}\left[2^{1 / 3} \frac{\mathrm{Ai}^{\prime}\left(2^{1 / 3} t\right)}{\operatorname{Ai}\left(2^{1 / 3} t\right)}-2^{1 / 3} \frac{\mathrm{Ai}^{\prime}(0)}{\operatorname{Ai}(0)}\right] & =\frac{\pi}{\sin \pi s} 2^{1 / 3(1-s)} \Lambda(1-s), \\
\mathcal{M}\left[G\left(2^{-3 / 2} t\right)\right] & =3 \sqrt{\pi} 2^{s / 2} \Gamma\left(\frac{3}{2}+\frac{3}{2} s\right) \Lambda\left(\frac{3}{2}+\frac{3}{2} s\right), \\
\mathcal{M}\left[2^{-3 / 2} w\left(2^{-3 / 2} t\right)\right] & =3 \sqrt{2 \pi} 2^{-s / 2} \frac{\Gamma\left(3-\frac{3}{2} s\right)}{\Gamma(1-s)} \Lambda\left(3-\frac{3}{2} s\right) .
\end{aligned}
$$

In particular, the moments of the Airy distribution exist for any $s \in \mathbb{C}$ and satisfy

$$
\mathbf{E}\left[\left(\frac{\mathcal{A}}{\sqrt{8}}\right)^{s}\right]=3 \sqrt{\pi} 2^{-s / 2} \frac{\Gamma\left(\frac{3}{2}(1-s)\right)}{\Gamma(-s)} \Lambda\left(\frac{3}{2}(1-s)\right),
$$

where $\Lambda\left(\frac{3}{2}(1-s)\right)$ is to be taken either as the power sum symmetric function of the $\alpha_{k}$ by (25) for $\Re(s)<0$, or as one of the analytic continuation forms of Lemma 2 for $\Re(s) \geq 0$.

PROOF. The proof of (29) starts with the fact that the Airy function is an entire function whose growth at $\infty$ is $O\left(e^{2 / 3|z|^{3 / 2}}\right)$, so that it is of order $\frac{3}{2}$. On the other hand, its zeros are such that

$$
\sum_{k} \frac{1}{\alpha_{k}}=+\infty, \quad \sum_{k} \frac{1}{\alpha_{k}^{2}}<+\infty
$$

meaning that it has genus 1 . Consequently, the Weierstrass-Hadamard factorization theorem [19, Chapter 15] applies and one has

$$
\operatorname{Ai}(z)=e^{d_{0}+d_{1} z} \prod_{k \geq 0}\left(1+\frac{z}{\alpha_{k}}\right) e^{-z / \alpha_{k}},
$$

for constants $d_{0}, d_{1}$ that are identified by the expansion of $\operatorname{Ai}(z)$ at 0 :

$$
d_{0}=\log \operatorname{Ai}(0), \quad d_{1}=\frac{\operatorname{Ai}^{\prime}(0)}{\operatorname{Ai}(0)} .
$$

Accordingly, the Mittag-Leffler decomposition of the logarithmic derivative of $\operatorname{Ai}(z)$ deduced from (33) is

$$
\frac{\operatorname{Ai}^{\prime}(z)}{\operatorname{Ai}(z)}-\frac{\operatorname{Ai}^{\prime}(0)}{\operatorname{Ai}(0)}=\sum_{k \geq 1}\left(\frac{1}{z+\alpha_{k}}-\frac{1}{\alpha_{k}}\right) .
$$

This form is a harmonic sum whose Mellin transform is

$$
\mathcal{M}\left[\frac{\mathrm{Ai}^{\prime}(z)}{\operatorname{Ai}(z)}-\frac{\operatorname{Ai}^{\prime}(0)}{\operatorname{Ai}(0)}\right]=\Lambda(1-s) \frac{\pi}{\sin \pi s},
$$


by virtue of the harmonic sum property (23) and the basic transform of $1 /(1+z)$ that equals $\pi / \sin \pi s$. Thus, (29) is established for $\Re(s) \in\left(-1,-\frac{1}{2}\right)$.

Next, we observe that, by the harmonic integral property (24), the Mellin transforms of $w$ and $G$ are related by

$$
w^{*}(s)=\frac{G^{*}(1-s)}{\Gamma(1-s)},
$$

so that it suffices to evaluate $G^{*}(s)$. The transform of $G$ is itself obtained by applying the harmonic sum property (23) to the exponential sum (15). (Equivalently, one could apply the harmonic integral property (24) to the Laplace-like transform (14).)

First, the three Mellin transforms above are meromorphic in the whole of the complex plane, given our earlier discussion of $\Lambda(s)$. The transform (29) is analytic for $\Re(s) \in$ $\left(-1,-\frac{1}{2}\right)$, while the transform of $G$ is analytic in $\Re(s)>0$. The transform of $w$ is a priori meromorphic in the whole of $\mathbb{C}$. It represents in fact a function analytic in the whole of $\mathbb{C}$ since the only poles of $\Lambda(s)$ which are at $s=\frac{3}{2},-\frac{3}{2},-\frac{9}{2}, \ldots$ are cancelled by the gamma function in the denominator of (31).

Finally, the Mellin transform of the density, corresponding to the right of (31), is Lebesgue integrable on any vertical line since it is a product of a rational form in gamma functions that decreases exponentially towards $\pm i \infty$ and of a $\Lambda$ factor that is only of polynomial growth by Lemma 2 . Thus, by a version of Mellin inversion (see Theorem 29 on p. 46 of [25]), the Mellin integral expressing $\mathcal{M}\left[2^{-3 / 2} w\left(2^{-3 / 2} t\right)\right]$ does converge for every $s$. The moment formula (32) is then plainly the translation of (31) by $s \mapsto s+1$.

The last property stated in (32) corresponds to the existence of moments of all positive as well as negative orders for the Airy distribution, a fact that is characteristic of fast decaying tails both at 0 and at $+\infty$.

4. Moments of Negative Order. As already pointed out in (22), the Mellin transform of a density function evaluated at $s$ is none other than the moment of order $s-1$ of the corresponding random variable. In the case of the Airy distribution, the Mellin transform is expressed in terms of the root zeta function $\Lambda(s)$ of $\mathrm{Ai}(z)$. Thus, any explicit value of the root zeta function provides an explicit moment evaluation. We proceed with this programme and determine here some of the negative moments in connection with the expansion of $\operatorname{Ai}(z)$ at 0 corresponding to values of $\Lambda(s)$ with $s>0$.

THEOREM 3 (Moments of Negative Order). Let $m$ be any real number such that $m>0$. Then the moment of order $-m$ of the Airy distribution is expressible in terms of the root zeta function $\Lambda$ as given by (25) in its region of convergence:

$$
E\left[\left(\frac{\mathcal{A}}{\sqrt{8}}\right)^{-m}\right]=3 \sqrt{\pi} 2^{m / 2} \frac{\Gamma\left(\frac{3}{2}+\frac{3}{2} m\right)}{\Gamma(m)} \Lambda\left(\frac{3}{2}+\frac{3}{2} m\right) .
$$


Furthermore, the moments of odd negative order $-m=-1-2 p$ for integers $p \geq 0$ are rationally expressible in terms of $\sqrt{\pi}, \Gamma\left(\frac{1}{3}\right)$, and algebraic radicals. One has

$$
E\left[\left(\frac{\mathcal{A}}{\sqrt{8}}\right)^{-1-2 p}\right]=-3 \sqrt{2 \pi} 2^{p} \frac{\Gamma(3+3 p)}{\Gamma(1+2 p)}(-1)^{3 p}\left[z^{2+3 p}\right] \frac{\mathrm{Ai}^{\prime}(z)}{\operatorname{Ai}(z)},
$$

where the bracket notation $\left[z^{a}\right] f(z)$ refers to the coefficient of $z^{a}$ in the analytic expansion of $f(z)$ at 0 . For instance, one has

$$
\begin{aligned}
& E\left[\left(\frac{\mathcal{A}}{\sqrt{8}}\right)^{-1}\right]=\sqrt{2 \pi}\left(3-16 \frac{\pi^{3} \sqrt{3}}{\Gamma\left(\frac{1}{3}\right)^{6}}\right), \\
& E\left[\left(\frac{\mathcal{A}}{\sqrt{8}}\right)^{-3}\right]=\sqrt{2 \pi}\left(18-480 \frac{\pi^{3} \sqrt{3}}{\Gamma\left(\frac{1}{3}\right)^{6}}+7680 \frac{\pi^{6}}{\Gamma\left(\frac{1}{3}\right)^{12}}\right) .
\end{aligned}
$$

PROOF. The first equation of the statement is a direct rephrasing of the Mellin relation (31). Note that for moments of order $-m<0$, the sum (25) over Airy zeros is convergent, so that moments can be estimated directly by (25) in all cases.

For the second equation, the Mittag-Leffler expansion (34) entails, upon expanding $1 /(z+\alpha)$ and exchanging summations,

$$
\frac{\operatorname{Ai}^{\prime}(z)}{\operatorname{Ai}(z)}-\frac{\operatorname{Ai}^{\prime}(0)}{\operatorname{Ai}(0)}=\sum_{k \geq 1} \Lambda(1+k)(-z)^{k} .
$$

Thus, the values of $\Lambda$ at the positive integers are explicit, given the classical expansion of $\operatorname{Ai}(z)$ at 0 that starts like

$$
\operatorname{Ai}(z)=\frac{1}{6} \frac{3^{5 / 6} \Gamma\left(\frac{1}{3}\right)}{\pi}-\frac{1}{3} \frac{3^{2 / 3}}{\Gamma\left(\frac{1}{3}\right)} z+\frac{1}{36} \frac{3^{5 / 6} \Gamma\left(\frac{1}{3}\right)}{\pi} z^{3}-\frac{1}{36} \frac{3^{2 / 3}}{\Gamma\left(\frac{1}{3}\right)} z^{4}+\cdots
$$

and it involves rationally $\pi$ and $\Gamma\left(\frac{1}{3}\right)$ in addition to algebraic radicals. This leads to

$$
\frac{\mathrm{Ai}^{\prime}(z)}{\operatorname{Ai}(z)}=-\frac{2}{3} \frac{\pi 3^{5 / 6}}{\Gamma\left(\frac{1}{3}\right)^{2}}-\frac{4}{3} \frac{\pi^{2} 3^{2 / 3}}{\Gamma\left(\frac{1}{3}\right)^{4}} z+\frac{1}{6} \frac{\left(3^{5 / 6} \Gamma\left(\frac{1}{3}\right)^{6}-16 \cdot 3^{1 / 3} \pi^{3}\right) 3^{1 / 6}}{\Gamma\left(\frac{1}{3}\right)^{6}} z^{2}+\cdots,
$$

which in turn gives, by way of (36) and (38), the moments in (37).

The proof also gives access to certain moments of order a multiple of $-\frac{1}{3}$.

THEOREM 4 (Moments of Fractional Negative Orders). The moments of negative fractional orders

$$
-\frac{5}{3},-\frac{7}{3},-\frac{11}{3},-\frac{13}{3}, \ldots
$$

evaluate in closed form by (37); for instance,

$$
E\left[\left(\frac{\mathcal{A}}{\sqrt{8}}\right)^{-5 / 3}\right]=\frac{9 \sqrt{\pi} 2^{5 / 6}}{\Gamma\left(\frac{1}{3}\right)^{7}}\left(3^{1 / 3} \Gamma\left(\frac{1}{3}\right)^{6}-8 \cdot 3^{5 / 6} \pi^{3}\right) .
$$

Thus, moments of negative order are closely related to the expansion of $\operatorname{Ai}(z)$ at 0 . 
5. Moments of Positive Order. We continue with the programme outlined in the previous section and examine the consequences of the root zeta function expression (32) of Proposition 3. The moments of positive order are by definition given by the asymptotic behaviour of the Airy function $\operatorname{Ai}(z)$ at $+\infty$. Here, we show that such moments are also indirectly determined by the behaviour of $\operatorname{Ai}(z)$ towards $-\infty$, where the function oscillates rapidly, this via the coefficients in the asymptotic expansion of the zeros $-\alpha_{k}$.

Consider the function

$$
h(x):=\frac{\operatorname{Ai}^{\prime}(x)}{\operatorname{Ai}(x)}-\frac{\operatorname{Ai}^{\prime}(0)}{\operatorname{Ai}(0)}
$$

As $x \rightarrow \infty$, one has

$h(x) \sim-x^{1 / 2}-\frac{\mathrm{Ai}^{\prime}(0)}{\mathrm{Ai}(0)}-\frac{1}{4} x^{-1}+\frac{5}{32} x^{-5 / 2}-\frac{15}{64} x^{-4}+\frac{1105}{2048} x^{-11 / 2}-\frac{1695}{1024} x^{-7}+\cdots$.

The Mellin transform of $h(x)$ exists a priori in the fundamental strip $-1<\Re(s)<-\frac{1}{2}$ where it equals (we repeat here (35))

$$
h^{*}(s)=\frac{\pi}{\sin \pi s} \Lambda(1-s),
$$

and $\Lambda(s)$ is already known to be meromorphically continuable (with simple poles at most) to the whole of $\mathbb{C}$. Finally, the mapping property of Mellin transforms implies that the singular expansion of $h^{*}(s)$ is the image of the asymptotic expansion of $h(x)$ at $+\infty$ :

$$
h^{*}(s) \asymp\left(\frac{\operatorname{Ai}^{\prime}(0)}{\operatorname{Ai}(0)} \frac{1}{s}\right)_{s=0}-\sum_{r \geq 0}\left((-1)^{r} \frac{\Omega_{r}}{2^{r} r !} \frac{1}{s-(3 r-1) / 2}\right)_{s=(3 r-1) / 2}
$$

(Such an expansion is nothing but the formal collection of all singular parts at all singularities, here for $\Re(s)>-\frac{1}{2}$, and it results from the direct version of the "mapping property".)

Special values of $\Lambda(s)$ on the positive line (that is, in a region where the sums (27) over Airy zeros that express it involve convergence adjustments) result from a closer examination of the expansion (41), as we now explain.

We start with the first two terms of (41).

- At $s=-\frac{1}{2}$, one has

$$
\Lambda(1-s) \frac{\pi}{\sin \pi s} \sim \frac{1}{s+\frac{1}{2}},
$$

which corresponds to the principal part of $\Lambda$ at the simple pole $\frac{3}{2}$, reflecting the asymptotic equivalence $\alpha_{k} \sim(3 \pi k / 2)^{2 / 3}$.

- The value of $\Lambda$ (1) results from comparing the residues at $s=1$ in (40) and (41):

$$
\Lambda(1)=\frac{\operatorname{Ai}^{\prime}(0)}{\operatorname{Ai}(0)}=-\frac{2}{3} 3^{5 / 6} \frac{\pi}{\Gamma\left(\frac{1}{3}\right)^{2}} .
$$

This gives explicitly the moment of order $\frac{1}{3}$ that is related to $\Lambda(1)$. 
THEOREM 5 (Moment of Order One-Third). The moment of order $\frac{1}{3}$ of the Airy distribution is expressible in terms of $\Gamma\left(\frac{1}{3}\right)$ as well as in terms of Airy zeros as $\left(\rho=(3 \pi / 2)^{2 / 3}\right)$

$$
\begin{aligned}
E\left[\left(\frac{\mathcal{A}}{\sqrt{8}}\right)^{1 / 3}\right] & =2^{-1 / 6} 3^{1 / 3} \frac{\sqrt{\pi}}{\Gamma\left(\frac{1}{3}\right)} \\
& =-\frac{2^{5 / 6}}{3^{1 / 2} \sqrt{\pi}} \Gamma\left(\frac{1}{3}\right)\left[\frac{\zeta\left(\frac{2}{3}\right)}{\rho}+\sum_{k}\left(\frac{1}{\alpha_{k}}-\frac{1}{\rho k^{2 / 3}}\right)\right] .
\end{aligned}
$$

The alternative expression with Airy zeros is an application of Lemma 2 and specifically of (27). Numerically, taking the first 1000 Airy zeros yields an accuracy of about $10^{-3}$ in the second formula.

The authors communicated a preliminary version of the present note to Marc Yor in December 1999. With astounding speed, Yor observed that the evaluation of the moment of order $\frac{1}{3}$ can be derived rather simply from the Bessel bridge model of the Brownian excursion and he refers to Theorem 3.5 of Chapter XI in his book [18].

We finally examine the effect of the other singularities in the expansion (41) of $h^{*}(s)$. The function $\pi / \sin \pi s$ is singular at $s=0,1,2,3, \ldots$ and it has residue $(-1)^{m}$ at $s=m$. Thus, comparison of the poles dictated by (40) and (41) shows that the interesting points on the positive real line are those of

$$
\{1,2,3,4, \ldots\} \cup\left\{1, \frac{5}{2}, 4, \frac{11}{2}, \ldots\right\} .
$$

These sets are not disjoint: the first one corresponds to the singularities of $\pi / \sin \pi s$, while the second one represents potential singularities of $\Lambda(s)$. Because of overlaps, several cases are to be distinguished:

(i) For $s \in S_{1}$, where $S_{1}=\{2,3,5,6,8,9, \ldots\}$, there is no pole of $h^{*}(s)$ and, since $\pi / \sin \pi s$ is singular, one must have

$$
\Lambda(-1)=\Lambda(-2)=\Lambda(-4)=\Lambda(-5)=\cdots=0 .
$$

This corresponds to an explicit evaluation of certain sums over Airy zeros in terms of values of the zeta function.

(ii) For $s \in S_{2}$, where $S_{2}=\{1,4,7,10, \ldots\}, \pi / \sin \pi s$ has a simple pole with residue \pm 1 , so that $\Lambda(1-s)$ must have a finite nonzero value,

$$
\Lambda(-3 m)=\frac{\Omega_{2 m+1}}{2^{2 m+1}(2 m+1) !} .
$$

This corresponds to a relation between moments of odd order of the Airy distribution and sums over Airy zeros.

(iii) For $s \in S_{3}$, where $S_{3}=\left\{\frac{5}{2}, \frac{11}{2}, \frac{17}{2}, \ldots\right\}$, the function $\pi / \sin \pi s$ has value $\pm \pi$, and the singularity of $h^{*}(s)$ must arise from a simple pole of $\Lambda(1-s)$ there. Identification of residues with (41) yields

$$
\left.\operatorname{Res} \Lambda(s)\right|_{s=3(1 / 2-m)}=(-1)^{m-1} \frac{\Omega_{2 m}}{\pi 2^{2 m}(2 m) !} \quad \text { for } \quad m \geq 1 .
$$


This corresponds to a relation between moments of even order of the Airy distribution and residues of $\Lambda$ that themselves, in accordance with (27), relate to the coefficients in the asymptotic expansion (20) of the $\alpha_{k}$.

As a consequence of this discussion, we obtain:

THEOREM 6 (Moments of Positive Order). Let $m$ be any real number satisfying $m>0$ and $m \neq 2,4,6, \ldots$. The moment of order $m$ of the Airy distribution is expressible in terms of sums over Airy zeros with convergence factors in the sense of Lemma 2:

$$
\mathbf{E}\left[\left(\frac{\mathcal{A}}{\sqrt{8}}\right)^{m}\right]=3 \sqrt{\pi} 2^{-m / 2} \frac{\Gamma\left(\frac{3}{2}(1-m)\right)}{\Gamma(-m)} \Lambda\left(\frac{3}{2}(1-m)\right) .
$$

The moments of even order $m=2,4,6, \ldots$ are expressible as finite polynomial forms in the coefficients $\left\{a_{i}\right\}$ in the expansion (21) of the $\alpha_{k}$.

PROOF. The first statement follows directly from Proposition 3, (32), and from the effective formulae of the continuation lemma, Lemma 2. The second statement is a rephrasing of Case (iii) of the discussion above.

EXAMPLES. As an illustration of Case (i), we have $\Lambda(-1)=0$, which, thanks to the continuation formula of Lemma 2 taken with $q=1$ gives the evaluation

$$
\sum_{k}\left[\alpha_{k}-\rho k^{2 / 3}+\frac{\rho}{6} k^{-1 / 3}\right]=-\rho \zeta\left(-\frac{2}{3}\right)+\frac{\rho}{6} \zeta\left(\frac{1}{3}\right) .
$$

Case (ii) leads to similar evaluations but with an inhomogeneous term that involves the moments of odd order. Such evaluations are special cases of explicit evaluations of symmetric functions of zeros of entire functions of genus 1. (For instance, the series expansion of the logarithmic derivative of $\sin z$ gives rise to the evaluation of the zeta function at even integers by essentially similar devices.)

Case (iii) has a more curious feature: it relates directly the moments of even order to the asymptotic expansion of the Airy zeros. For instance, by (44), the "echo" of $\Omega_{2}=\frac{5}{4}$ is to be found in the fact that

$$
\Lambda(s) \sim \frac{5}{32 \pi} \frac{1}{s+\frac{3}{2}}, \quad s \rightarrow-\frac{3}{2},
$$

while the principal part of $\Lambda(s)$ is, in the notations of Lemma 2,

$$
\rho^{-3 / 2} g_{2}\left(-\frac{3}{2}\right) \zeta\left(\frac{2}{3} s+2\right) \sim \frac{3}{2} \rho^{-3 / 2} g_{2}\left(-\frac{3}{2}\right) \frac{1}{s+\frac{3}{2}},
$$

and $g_{2}(s)$ is itself determined by the coefficients $a_{1}, a_{2}$ in the asymptotic expansion (20) of $\alpha_{k}$. A closer look at the dependencies shows that moments of positive even order and coefficients in the asymptotic expansion of Airy zeros determine one another. ${ }^{4}$

\footnotetext{
${ }^{4}$ These relations can also be understood in the light of the fact that the expansion of the Airy zeros is fully determined by the oscillating expansion (19) of $\mathrm{Ai}(z)$, which, given general principles of linear differential equations, is essentially a formal variant of the expansion (1) at $+\infty$. The striking similarity of coefficients in (19) and (12) tells a good deal of the story.
} 
6. Postscript. The Airy distribution can be approached from many angles. As stated in the Introduction, we decided to explore here the direct consequences of the recursive definition of moments, a point of view that may be useful in combinatorial applications. A related motivation is the fact that a growing number of distributions in analytic combinatorics are found to involve the Airy function and a better understanding of the driving analysis is needed; see for instance the recent work [3] for an Airy distribution of the "map type" that describes the size of largest multiconnected components in random maps.

It is a fact that the appearance of the Airy distribution (of the area type) is a good indication of the possibility of a stronger functional limit theorem expressing convergence to the Brownian excursion process. That such is the case, by design, for discrete random walks (via the Brownian excursion area) and stochastic convergence has been determined recently for several of the examples mentioned in the Introduction. Also, as pointed out in relation to the evaluation of the moment of order $\frac{1}{3}$ and Yor's comments, alternative probabilistic derivations of some (or all?) of our results are possible.

What we feel to be perhaps the most striking outcome of our investigations is the dependency between complex analytic properties of the Airy function and probabilistic properties of the Airy distribution. A final summary is as follows:

\begin{tabular}{|c|c|}
\hline Airy function & Airy distribution \\
\hline $\mathrm{Ai}(z)$ as $z \rightarrow+\infty$ & $\begin{array}{l}\text { Positive integral moments (Definition } 1 \text { and Proposition 1) } \\
\quad-\text { Moment of order } \frac{1}{3} \text { (explicit) }\end{array}$ \\
\hline $\mathrm{Ai}(z)$ as $z \rightarrow 0$ & $\begin{array}{l}\text { Negative moments } \\
\quad \text { - Moments of order }-1,-3,-5 \text {, etc. (explicit) } \\
\text { - Moments of order }-\frac{5}{3},-\frac{7}{3},-\frac{11}{3} \text {, etc. (explicit) }\end{array}$ \\
\hline $\begin{array}{l}\operatorname{Ai}(z) \text { as } z \rightarrow-\infty \\
\quad\left(\text { Airy zeros }-\alpha_{k}\right)\end{array}$ & $\begin{array}{l}\text { All moments via the root zeta function } \Lambda(s) \\
\text { - Moments of order } 2,4,6 \text {, etc. and asymptotic } \\
\text { exponential of } \alpha_{k}\end{array}$ \\
\hline
\end{tabular}

Acknowledgment. The authors are grateful to Marc Yor for many insightful comments on a preliminary draft of this paper.

\section{References}

[1] M. Abramowitz and I. A. Stegun. Handbook of Mathematical Functions. Dover, New York, 1973. A reprint of the tenth National Bureau of Standards edition, 1964.

[2] D. Aldous and J. Pitman. The standard additive coalescent. The Annals of Probability, 26(4):1703-1726, 1998.

[3] C. Banderier, P. Flajolet, G. Schaeffer, and M. Soria. Planar maps and Airy phenomena. In E. Welzl, U. Montanari, and J. Rolim, editors, Automata, Languages, and Programming, Number 1853 in Lecture Notes in Computer Science, pages 388-402. Springer-Verlag, Berlin, 2000. Proceedings of the 27th ICALP Conference, Geneva, July 2000.

[4] P. Billingsley. Probability and Measure, 2nd edition. Wiley, New York, 1986.

[5] P. Chassaing and J.-F. Marckert. Parking functions, empirical processes and the width of rooted labeled trees. Electronic Journal of Combinatorics, 8, R14, 2001. 18 pages. 
[6] M. Csőrgő, Z. Shi, and M. Yor. Some asymptotic properties of the local time of the uniform empirical processes. Bernoulli, 5(6):1035-1058, December 1999.

[7] G. Doetsch. Handbuch der Laplace Transformation, Vols. 1-3. Birkhäuser Verlag, Basel, 1955.

[8] P. Duchon. Q-grammars and wall polyominoes. Annals of Combinatorics, 3:311-321, 1999.

[9] P. Flajolet. Combinatorial aspects of continued fractions. Discrete Mathematics, 32:125-161, 1980.

[10] P. Flajolet, X. Gourdon, and P. Dumas. Mellin transforms and asymptotics: Harmonic sums. Theoretical Computer Science, 144(1-2):3-58, June 1995.

[11] P. Flajolet, P. Poblete, and A. Viola. On the analysis of linear probing hashing. Algorithmica, 22(4):490515, December 1998.

[12] B. Gittenberger and G. Louchard. The Brownian excursion multi-dimensional local time density. Journal of Applied Probability, 36(2):350-373, 1999.

[13] S. Janson, D. E. Knuth, T. Luczak, and B. Pittel. The birth of the giant component. Random Structures \& Algorithms, 4(3):233-358, 1993.

[14] P. Kirschenhofer, H. Prodinger, and R. Tichy. Über einige Funktionaldifferentialgleichungen aus der Analyse von Algorithmen. In E. Hlawka, editor, Zahlentheoretische Analysis II, Number 1262 in Lecture Notes in Mathematics, pages 111-123. Springer-Verlag, Berlin, 1987.

[15] D. E. Knuth. The Art of Computer Programming, Volume 2: Seminumerical Algorithms, 3rd edition; Addison-Wesley, 1998.

[16] G. Louchard. The Brownian excursion: a numerical analysis. Computers and Mathematics with Applications, 10(6):413-417, 1984

[17] G. Louchard. Kac's formula, Lévy's local time and Brownian excursion. Journal of Applied Probability, 21:479-499, 1984

[18] D. Revuz and M. Yor. Continuous Martingales and Brownian Motion. Springer-Verlag, Berlin, 1991.

[19] L. A. Rubel. Entire and Meromorphic Functions. Springer-Verlag, New York, 1996.

[20] L. A. Shepp. On the integral of the absolute value of the pinned Wiener process. Annals of Probability, 10:234-239, 1982

[21] J. Spencer. Enumerating graphs and Brownian motion. Communications on Pure and Applied Mathematics, 50:293-296, 1997.

[22] L. Takács. A Bernoulli excursion and its various applications. Advances in Applied Probability, 23:557-585, 1991.

[23] L. Takács. Conditional limit theorems for branching processes. Journal of Applied Mathematics and Stochastic Analysis, 4(4):263-292, 1991.

[24] L. Takács. On a probability problem connected with railway traffic. Journal of Applied Mathematics and Stochastic Analysis, 4(1):1-27, 1991.

[25] E. C. Titchmarsh. Introduction to the Theory of Fourier Integrals. Chelsea, New York, 1986. Reprinted from the second edition, Oxford University Press, Oxford, 1948.

[26] R. Wong. Asymptotic Approximations of Integrals. Academic Press, New York, 1989.

[27] E. M. Wright. The number of connected sparsely edged graphs. Journal of Graph Theory, 1:317-330, 1977. 